\title{
Electronic Structures and Noncollinear Magnetic Properties of Structurally Disordered Fe
}

\author{
Jin Ho Park and B. I. Min* \\ Department of Physics, Pohang University of Science and Technology, Pohang, Gyungbuk 790-784, Korea
}

(Received 2 November 2009, Received in final form 3 February 2010, Accepted 3 February 2010)

\begin{abstract}
The magnetic properties of amorphous Fe were investigated by examining the electronic structures of structurally disordered Fe systems generated from crystalline bec and fec Fe using a Monte-Carlo simulation. As a rst principles band method, the real space spin-polarized tight-binding linearized-mun-tin-orbital recursion method was used in the local spin density approximation. Compared to the crystalline system, the electronic structures of the disordered systems were characterized by a broadened band width, smoothened local density of states, and reduced local magnetic moment. The magnetic structures depend on the short range configurations. The antiferromagnetic structure is the most stable for a bcc-based disordered system, whereas the noncollinear spin spiral structure is more stable for a fec-based system.
\end{abstract}

Keywords : electronic structure of disordered solids, amorphous Fe, amorphous magnetic materials

\section{Introduction}

Amorphous alloy systems have very different magnetic characteristics compared to crystalline systems [1,2]. In the case of amorphous $\mathrm{Fe}(\mathrm{a}-\mathrm{Fe})$, the difficulties in making pure a-Fe and the metastability in a very small temperature range have resulted in experimental investigations being performed only on Fe-rich amorphous alloys containing a certain amount of metalloids (a-FeB, a-FeP, etc.) or other transition metals (a-FeZr, a-FeY, etc.). Various intriguing phases have been observed in Fe-rich amorphous alloys, such as the spin glass phase [3-5] and an asperomagnetic phase [6], etc. This suggests that amorphous alloys with a similar composition to pure a-Fe have a noncollinear spin configuration. The magnetic states of amorphous alloys also depend on the preparation and thermal treatment of the samples. Amorphous $\mathrm{Fe}_{2} \mathrm{Y}$ prepared by melt-spinning becomes a ferromagnet [7], whereas $\mathrm{a}-\mathrm{Fe}_{2} \mathrm{Y}$ prepared by sputtering becomes a concentrated spin-glass [8]. Therefore, it is unclear how the structural disorder affects the magnetic state in amorphous alloys.

The different behaviors of a-Fe alloys have been attributed tentatively to fluctuations in the distance and

*Corresponding author: Tel: +82-54-279-2074

Fax: +82-54-279-3099, e-mail: bimin@postech.ac.kr number of the Fe coordinations [9] as well as to the different initial conditions in fabricating the alloys [10]. A few theoretical studies based on the model Hamiltonians have been reported $[11,12]$. However, the results are not consistent with the experimental data. Therefore, rstprinciples electronic structure calculations that consider the noncollinear spin congurations are required. Firstprinciples band structure calculations are very complicated and time-consuming because there is no proper translational symmetry in an amorphous system. Furthermore, it is almost impossible to simulate a spin-glass state with a noncollinear spin structure as found in Fe-rich amorphous alloys.

There are several reports using the rst principles band calculations in a local-spin-density approximation (LSDA). Hafner et al. [13] carried out self-consistent tight-binding linear-muffin-tin-orbital (TB-LMTO) [14] band calculations for a supercell consisting of 64 atoms by assuming the collinear spin configuration. They reported that under compression, isolated local moments tend to be flipped down to the opposite direction. Lorenz and Hafner $[15,16]$ examined the noncollinear spin structure of itinerant magnets using the Hubbard Hamiltonian constructed by the TB-LMTO. They showed that a-Fe undergoes a continuous transition from a ferromagnetic to a spin-glasslike state with increasing density. They assumed an effective universal Stoner parameter to obtain the spin polariz- 
ed electronic structure. Liebs et al. [17] studied structurally disordered $\mathrm{Fe}$, $\mathrm{Co}$ and $\mathrm{Ni}$ by treating the noncollinear magnetic structures in a fully self-consistent manner. Their results for $\mathrm{Fe}$ showed that with increasing density, the noncollinear structures are energetically lower than collinear ones. However, the calculation was not free of periodicity problems because of the small number of atoms (16 atoms) within the cell.

Real-space large cluster calculations have also been performed for a large amorphous cluster consisting of several hundreds of atoms. The real-space TB-LMTO method was rst applied to a-Fe in its simplest form by Fujiwara [18, 19]. The local density of states (LDOS) was obtained self-consistently by averaging over a few selected atoms in the central unit cell. A more detailed account of the method was reported by Nowak et al. [20] with the paramagnetic electronic structure calculation for a- $\mathrm{Fe}_{80} \mathrm{~B}_{20}$. Bratkovsky and Smirnov [21] performed a spin-polarized calculation for a ferromagnetic state of aFe. Krey et al. [22, 23] examined noncollinear magnetic structures in amorphous systems using the TB-LMTOrecursion method [24]. They used an empirical SlaterKoster [25] parameterization using a fitted value of the exchange parameter. They showed that for a-Fe, which is a noncollinear canted magnetic structure, either the asperomagnetic or speromagnetic state is energetically more stable.

This study investigated the ground state electronic and magnetic properties of structurally disordered $\mathrm{Fe}$, using the real space scheme of the TB-LMTO recursion method. The electronic structures are examined by varying the disorder of the cluster and by allowing the noncollinear polarized direction of the magnetic moment of each atom. Since a precise calculation of a completely disordered system is not possible, one has to resort to a simple model of an amorphous system. For this purpose, this study employed a 54-atom cluster generated from a bcc Fe and a 64-atom cluster generated from fcc $\mathrm{Fe}$, where all the atoms up to the third nearest neighbors from the central atom are included in the cluster. The calculations are almost free of periodicity problems because the TB-LMTO Hamiltonian considers atoms up to the third nearest neighbors for each site.

\section{Computational Method}

Real-space TB-LMTO recursion calculations for disordered Fe using the supercell approach. The real-space TB-LMTO method is a rst-principles and self-consistent band calculation based on the LMTO-ASA (atomicsphere approximation) formalism. It is similar to the conventional LMTO-ASA scheme except that the LDOS is obtained in real space using the recursion method rather than in k-space by solving the eigenvalue problem [14, $20,26]$. In the present study, the calculations were carried out with a LMTO basis of s, p, and d states, for which 31 pairs of recursion coefficients were evaluated. For a bccbased cluster, 9216 atoms were considered so that 31 levels of recursion coefficients could be calculated accurately.

The non-collinear spin structure was treated according to the scheme reported by Heine et al. [27, 28]. The Hamiltonian, $H_{R L, R^{\prime} L^{\prime}}$, is defined along the global $z$ axis, whereas the spin for each atom is arranged along the local $\xi_{R}$ axis. To perform the computations, the Hamiltonian must be expressed in terms of the local axis. Let us represent the Hamiltonian matrix elements $H_{R L \alpha, R^{\prime} L^{\prime} \beta}$ with respect to the global axes, and $H_{R L \mu, R^{\prime} L^{\prime} v}$ with respect to the local $\xi_{R}$ and $\xi_{R^{\prime}}$ axes. Then

$$
H_{R L \mu, R^{\prime} L^{\prime} v}=\left(D_{R}^{-1}\right)_{\mu \alpha} H_{R L \alpha, R^{\prime} L^{\prime} \beta}\left(D_{R^{\prime}}\right)_{\beta v}
$$

where $D_{R}$ is the rotation matrix[27];

$$
D_{R}= \pm\left[\begin{array}{cc}
\exp \left[-\frac{1}{2} i \varphi_{\dot{R}}\right] \cos \frac{1}{2} \theta_{R} & -\exp \left[\frac{1}{2} i \varphi_{\dot{R}}\right] \sin \frac{1}{2} \theta_{R} \\
\exp \left[-\frac{1}{2} i \varphi_{R}\right] \sin \frac{1}{2} \theta_{R} & \exp \left[\frac{1}{2} i \varphi_{\dot{R}}\right] \cos \frac{1}{2} \theta_{R}
\end{array}\right]
$$

Here angles $\theta_{R}$ and $\varphi_{R}$ define the direction of $\xi_{R}$ on the atom $\mathrm{R}$ relative to the global axis.

In this study, a one-electron Hamiltonian was constructed from (9216 atoms $\times 9$ orbitals $\times 2$ spin) states, and the LDOSs were calculated self-consistently for all sites (more than 50 sites) in several disordered unit cells. Therefore, it requires considerable computer time and memory size. A substantial amount of computer time is saved by setting all $\varphi_{R}=0$, because the Hamiltonian matrix then becomes real. Although it restricts the variety of congurations, one can still consider the physical situations by allowing to take on the full range from 0 to $2 \pi$. The spin configuration corresponding to the spin spiral state (all $\left.\varphi_{R}=0\right)$ is then considered, in which the spins within a plane are all parallel with polar angles $\theta_{R}$ with respect to the global axis and the polar angle increases from plane to plane.

Large supercells of 54 (bcc)-64 (fcc) atoms were used to simulate the amorphous structure. These cells were cut from a larger cluster of bcc or fcc crystalline $\mathrm{Fe}$, and relaxed under the periodic boundary condition through a Monte-Carlo scheme. A structurally disordered Fe was obtained by varying the amount of maximum displacement from the equilibrium position, $\delta R / R^{0}{ }_{N N}$, where $\delta R$ and 
$R_{N N}^{0}$ represent the displacement from the crystalline (equilibrium) position and the nearest neighbor (NN) distance of the crystalline Fe, respectively. At rst, a given number (54 or 64) of $\mathrm{Fe}$ atoms were placed into a cubic box on the sites of the bcc- or the fcc-lattice with a fixed lattice constant of $\mathrm{a}=2.78 \AA$ or $\mathrm{a}=3.58 \AA$. The atoms were then displaced in random directions, and the system was relaxed using the Monte-Carlo method under the periodic boundary condition. During the relaxation procedure, the volume of the cubic box and minimal nearest neighbor distance were fixed to $0.95 R^{0}{ }_{N N}$. The amorphous cell was repeated periodically, and for a bcc-based system, a cluster of 9216 atoms was then obtained by including all sites up to a certain distance from each atom of a central cube. The distance was chosen to guarantee that the coefficients in the 31 levels of the continued fraction were evaluated precisely.

\section{Results}

\subsection{Radial distribution function}

Since the exact positions of the atoms in amorphous materials are unknown, it was ensured that our cluster gives a reasonable radial distribution function when compared either to experiments or to more sophisticated calculations. The radial distribution function provides information on the short range local atomic configuration. Fig. 1 compares resulting radial distributions of the neighboring atoms from this model with the more sophisticated calculational result for a-Fe [29]. Both the fcc (Fig. 1(a)) and bcc-based disordered model (Fig. 1(c)) appear to reproduce the atomic configuration of a-Fe well (Fig. 1(b)). That is, the second crystalline peaks labeled B (fcc) and B (bcc) are smeared. Hence, the broad rst peaks labeled A and A' become predominant near $r_{1}=2.45 \AA$, which appears to be consistent with the rst dominant peak in a-Fe.

\subsection{Collinear magnetic structure}

The rst step to understand the magnetic properties in amorphous alloys was to examine their electronic structures. As the magnetic ground state of bcc Fe is ferromagnetic, the collinear ferromagnetic spin configuration was considered for bcc-based disordered Fe. Fig. 2 shows the LDOS for the bcc-based disordered Fe systems. In this calculation, the LDOSs of all atomic sites in the central unit cell are determined self-consistently. In previous TBLMTO calculations $[15,16,22,23]$, the LDOSs were determined by averaging over a few selected atoms in the central unit cell. The magnetic moments and LDOSs for disordered $\mathrm{Fe}$ are no longer homogeneous due to the structural disorder. The notable features in the LDOSs of

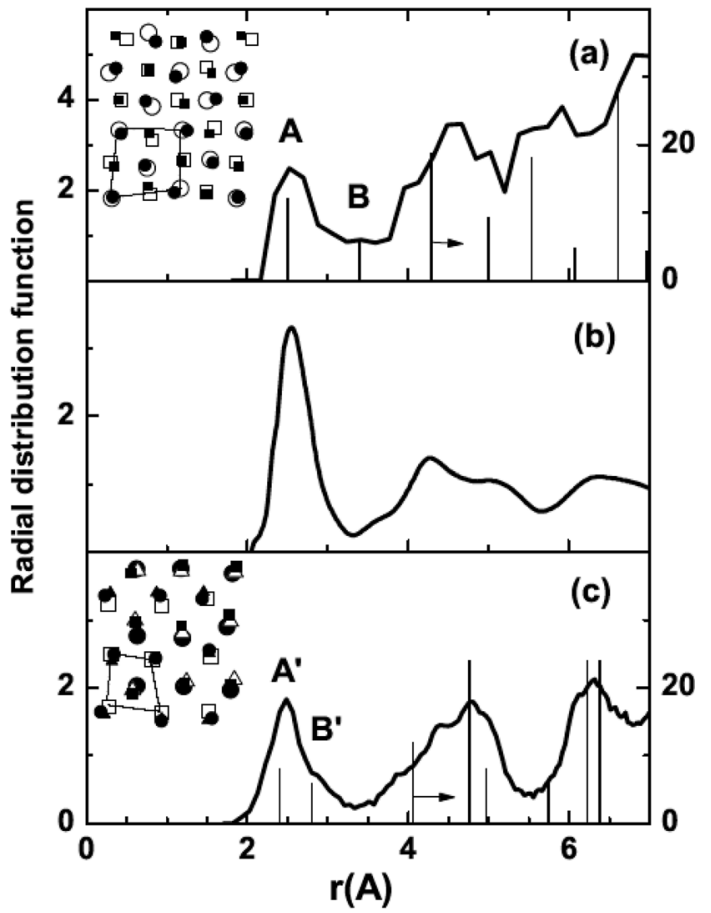

Fig. 1. The radial distribution functions for fcc- (a) and bccbased (c) amorphous systems, which are compared with the more sophisticated calculational result for a-Fe (b). The vertical bars referring to the right $y$-axis represent the number of atoms at the atomic positions in a crystalline system. The insets show the atomic congurations considered in the calculation. The different symbols represent the atoms in the different layers and the atoms connected by the line correspond to the ones in the basal plane.

the bcc-based a-Fe are: (i) the band width becomes wider than in crystalline bcc Fe, (ii) the main peak near the top of the majority spin band shifts toward the Fermi energy, and (iii) the band broadens with decreasing magnetic moment. That is, atoms with smaller magnetic moments have wider bands.

In Figs. 3(a) and 3(b), the magnetic moment behaviors were examined as a function of the disorderness $\delta R / R^{0}{ }_{N N}$ of the system and the sum of the inverse fifth power of the neighboring bond lengths, respectively. The disorderness increases with increasing displacement $\delta R$. Fig. 3(a) shows that in the bcc-based disordered $\mathrm{Fe}$ systems, the average magnetic moment decreases with increasing disorder. An atom with a closely-packed environment, i.e. having neighbors with reduced bond lengths, tends to have magnetic moment polarized antiferromagnetically to its neighboring spins. By introducing the disorder, the bond lengths become different from those of crystalline $\mathrm{Fe}$ but the average bond length is not changed due to the fixed volume. Instead, the average value of some power of the bond length is changed. Consequently, the hopping strength 


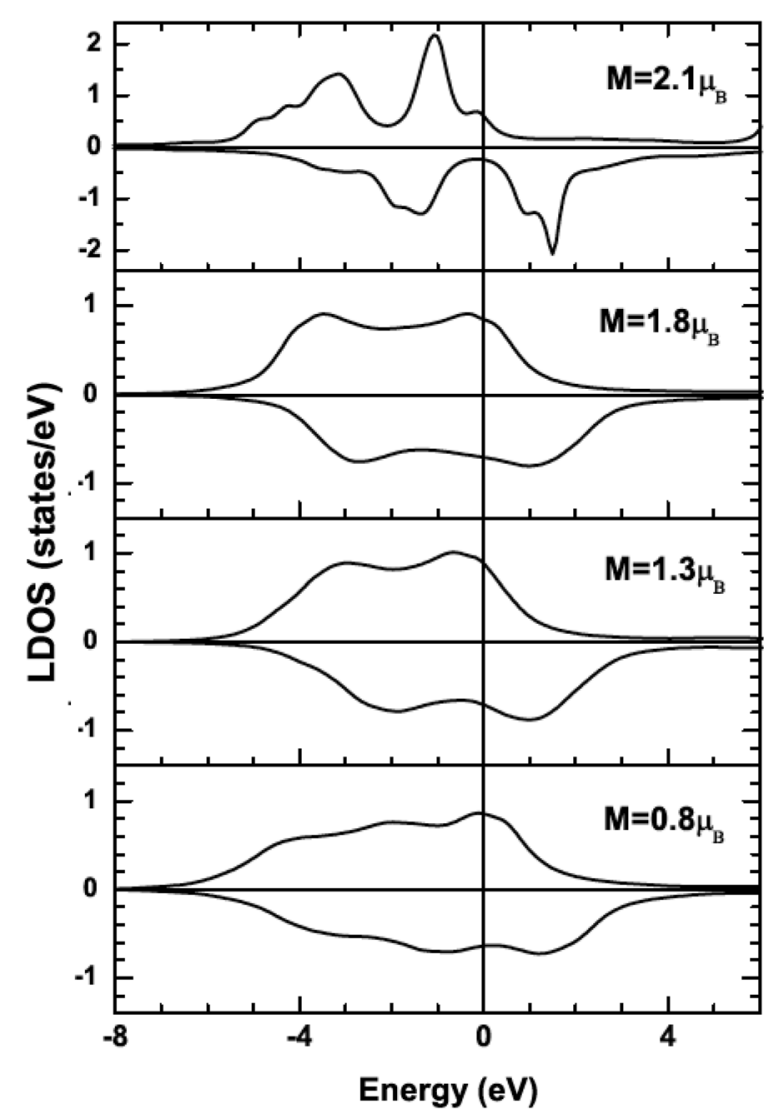

Fig. 2. LDOSs of the bcc-based disordered Fe at three different sites with different local magnetic moments, which are compared to the LDOS of the crystalline Fe given in the top panel. The ferromagnetic collinear spin configuration is assumed.
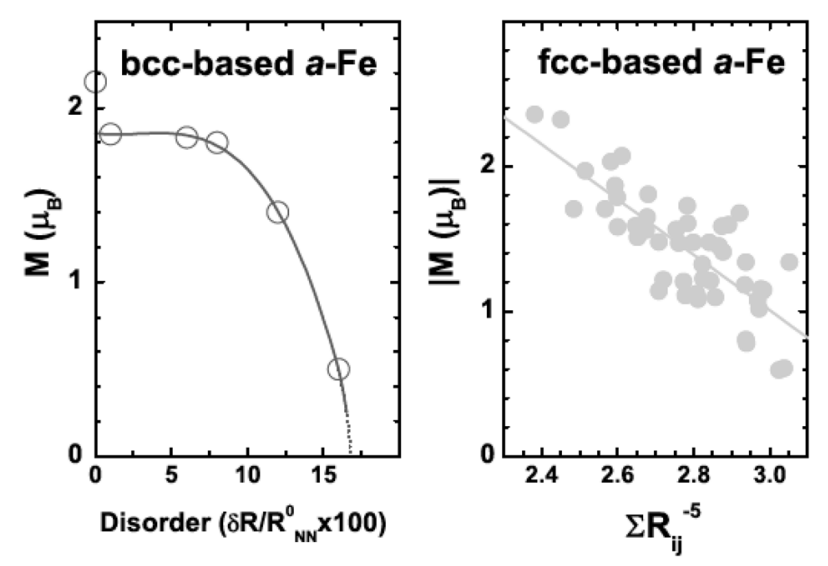

Fig. 3. (a) Average magnetic moments in the bcc-based disordered $\mathrm{Fe}$ as a function of the displacement of $\delta R$. (b) Local magnetic moments in the fcc-based disordered $\mathrm{Fe}$ as a function of the sum of the inverse fifth power of the bond lengths.

and band width would also be changed because they are proportional to the inverse power of the bond length. In the TB approximation, the hopping strength between the $\mathrm{d}$-states of the nearest neighbors is given by the inverse fifth power of the bond length $\left(\sim 1 / R^{5}\right)[24,25]$. Therefore, a decrease in the magnetic moment in the amorphous phase is expected to be associated with this change in hopping strength.

Indeed, as shown in Fig. 3(b), the local magnetic moment in the fcc-based disordered Fe decreases as a function of the sum of the inverse fifth power of the bond lengths. Since the magnetic ground state of crystalline fcc Fe is antiferromagnetic, the antiferromagnetic spin configuration for the fcc-based disordered Fe system in Fig. 3(b) was initially assumed. Although the data is scattered, one can recognize the almost linear relationship between the size of the local magnetic moment and the sum of the inverse fifth power of the bond lengths. These results suggest that the effective hopping strength increases in the disordered $\mathrm{Fe}$ to reduce the magnetic moment at each site.

The reason why the magnetic moment in the case of the bcc-based disordered system reduces so rapidly compared to the case of the fcc-based system was examined [30]. One can understand this behavior by referring to the radial distribution functions shown in Fig. 1. In the bccbased system, the second neighbor peak is located close to the rst peak, whereas in the fcc-based system, the second peak is located farther from the first peak. Therefore, in the bcc-based system, the effective number of nearest neighbors increases with increasing disorder, while, the number is relatively constant in the fcc-based system. That is, the effective number of nearest neighbors in the bcc-based system increases up to $\sim 10.5$, and the hopping strength increases accordingly to give rise to reduced magnetization.

The topological difference between the bcc and fccbased systems also needs to be considered. The magnetic moment variation in the FeZr series shows slowly increasing behavior with decreasing $\mathrm{Zr}$ concentration down to $14 \%$ [31]. Below this limit, the moment begins to decrease rapidly. This behavior was interpreted in terms of the appearance of two magnetic states of Fe below $14 \% \mathrm{Zr}$; ferromagnetic bcc $\mathrm{Fe}$ and antiferromagnetic fcc Fe. A similar behavior of the magnetic moment variation was observed in the FeB series [9]. It is likely that amorphous $\mathrm{FeB}$ varies from densely-packed fcc-like local order $\left(\mathrm{Fe}_{80} \mathrm{~B}_{20}\right)$ to a loosely-packed random structure with bcclike local order $\left(\mathrm{Fe}_{88} \mathrm{~B}_{12}\right)$. Both the $\mathrm{FeZr}$ and $\mathrm{FeB}$ series show rapid decreases in the magnetic moment and the Curie temperature in the bcc-like local order side, which is consistent with our interpretation.

\subsection{Noncollinear magnetic structure}

Consider the noncollinear spin spiral congurations for a- 


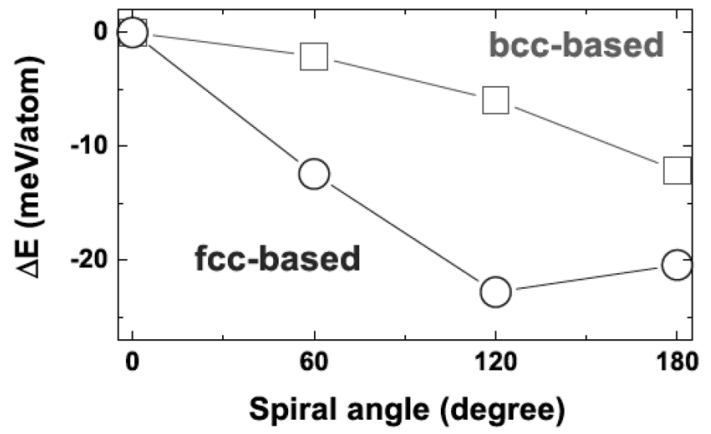

Fig. 4. Total energies of the bcc (squares) and fcc-based (circles) spin-noncollinear disordered systems as a function of the spiral angle between neighboring planes. The spiral angle of $0^{\circ}$ corresponds to the ferromagnetic arrangement. The disorderness, $\delta R / R^{0}{ }_{N N}$, for both systems are 0.1 .

Fe. Fig. 4 shows the total energy per atom as a function of the spiral angle between the neighboring planes for both bcc and fcc-based disordered Fe systems. Here, finite spiral angles of $0^{\circ}, 60^{\circ}, 120^{\circ}$ and $180^{\circ}$ were considered. In the bccbased disordered system, the antiferromagnetic spin configuration $\left(180^{\circ}\right)$ is the most stable, whereas in the fcc-based system, the noncollinear spin spiral configuration $\left(120^{\circ}\right)$ becomes more stable than the collinear ferromagnetic $\left(0^{\circ}\right)$ and antiferromagnetic $\left(180^{\circ}\right)$ congurations. The differences in total energy are in the order of $10 \mathrm{meV} / \mathrm{atom}$, which is 10 times larger than the results reported by Krey et al. who used empirical parameters [22, 23].

In the bcc-based disordered system, the magnetic moments of all the nearest neighbors of the central site are canted-polarized with an angle $\theta$. In contrast, in the fcc- based disordered system, the magnetic moments of the four nearest neighbors are polarized ferromagnetically with that of the central site, whereas those of eight nearest neighbors are canted-polarized with an angle $\theta$. This means that the atoms in the fcc-based disordered system have both ferromagnetic interactions within the plane and semi-antiferromagnetic interactions between the neighboring planes. The stable spin spiral phase in the fcc-based disordered system is expected to come from these combined ferromagnetic and antiferromagnetic interactions.

\section{Conclusion}

This study examined the electronic structures and magnetic properties of structurally disordered Fe systems using the spin-polarized TB-LMTO recursion band method in the LSDA. Compared to crystalline systems, the characteristics of the electronic structures of disordered systems are a broadened band width and smoothened LDOS. The increased band width reduces the local magnetic moments, and the various nearest neighbor hopping strengths at each site give rise to an inhomogeneous local magnetic moment distribution. The antiferromagnetic phase was found to be the most stable in the bcc-based disordered system, while the non-collinear spin spiral phase becomes more stable in the fcc-based system. The noncollinear spin configuration was induced not only by the inhomogeneous nearest neighbor magnetic interaction but also by the inhomogeneous hopping strengths between neighbors.

\section{Acknowledgments}

This study was supported by the NRF (Grant No.20090079947), and by the steel science project of POSCO.

\section{References}

[1] R. C. O'Handley, in Amorphous Metallic Alloys, edited by F. E. Luborsky, BMM, Butterworths, London (1983).

[2] M. L. Fdez-Gubieda, A. Garcia-Arribas, J. M. Barandiaran, R. L. Anton, I. Orue, P. Gorria, S. Pizzini, and A. Fontaine, Phys. Rev. B 62, 5746 (2000).

[3] N. Saito, H. Hiroyoshi, K. Fukamichi, and Y. Nakagawa, J. Phys. F 16, 911 (1986).

[4] J. M. D. Coey, D. Givord, A. Lienard, and J. P. Rebouillat, J. Phys. F 11, 2707 (1981).

[5] H. Wakabayashii, K. Fukamichi, H. Komatsu, T. Goto, T. Sakakibara, and K. Kuroda, in Proceedings of the International Symposium on Physics of Magnetic Materials 1986, World Scientic, Singapore (1987).

[6] H. Ma, H. P. Kunkel, and G. Williams, J. Phys.: Condens Matter 3, 5563 (1991).

[7] J. J. Croat and J. F. Herbst, J. Appl. Phys. 53, 2294 (1982).

[8] D. W. Forester, N. C. Koon, J. H. Schelleng, and J. J. Rhyne, J. Appl. Phys. 50, 7336 (1979).

[9] R. Hasegawa and R. Ray, J. Appl. Phys. 49, 4174 (1978).

[10] C. L. Chien, D. Musser, E. M. Gyorgy, R. C. Sherwood, H. S. Chen, F. E. Luborsky, and J. L. Walter, Phys. Rev. B 20, 283 (1979).

[11] Y. Kakehashi, Phys. Rev. B 43, 10820 (1991); T. Uchida and Y. Kakehashi, Phys. Rev. B 64, 54402 (2001).

[12] M. Yu and Y. Kakehashi, Phys. Rev. B 49, 15723 (1994).

[13] J. Hafner, M. Tegze, and Ch. Becker Phys. Rev. B 49, 285 (1994).

[14] O. K. Andersen and O. Jepsen, Phys. Rev. Lett. 53, 2571 (1984); O. Jepsen and M. Sob, in Electronic Band Structure and its Applications, edited by M. Yussou, Lecture Notes in Physics Vol. 283, Springer, Berlin (1987).

[15] R. Lorenz and J. Hafner, J. Magn. Magn. Mater. 139, 209 (1995).

[16] D. Spisak, J. Hafner, and R. Lorenz, J. Magn. Magn. Mater. 166, 303 (1997). 
[17] M. Liebs, K. Hummler, and M. Föhnle, Phys. Rev. B 51, 8664 (1995).

[18] T. Fujiwara, J. Phys. F: Met. Phys. 12, 661 (1982).

[19] T. Fujiwara, J. Non-Cryst. Solids 61 and 62, 1039 (1984).

[20] H. J. Nowak, O. K. Andersen, T. Fujiwara, O. Jepsen, and P. Vargas, Phys. Rev. B 44, 3577 (1991).

[21] A. M. Bratkovsky and A. V. Smirnov, Phys. Rev. B 48, 9606 (1993).

[22] U. Krey, S. Krompiewski, and U. Krauss, J. Magn. Magn. Mater. 86, 85 (1990).

[23] U. Krey, U. Krauss, and S. Krompiewski, J. Magn. Magn. Mater. 103, 37 (1992).

[24] R. Haydock, in Solid State Physics, edited by F. Seitz and D. Turnbull, Academic, New York (1980) Vol. 35, p.
215.

[25] J. C. Slater and G. F. Koster, Phys. Rev. 94, 1498 (1954).

[26] J. H. Park, S. K. Kwon, and B. I. Min, J. Korean Phys. Soc. 37, 109 (2000).

[27] M. V. You, V. Heine, A. J. Holden, and P. J. Lin-Chung, Phys. Rev. Lett. 44, 1282 (1980).

[28] M. V. You and V. Heine, J. Phys. F: Met. Phys. 12, 177 (1982).

[29] R. Yamamoto and M. Doyama, J. Phys. F 9, 617 (1979).

[30] The data for the fcc-based disordered system are not shown.

[31] S. Ohnuma, K. Shirakawa, M. Nose, and T. Masumoto, IEEE Trans. Magn. 16, 1129 (1980). 